\title{
A Novel Approach towards the Search for Gamma-ray Emission from the Northern Fermi Bubble with HAWC
}

\section{Pooja Surajbali ${ }^{a, *}$ on behalf of the HAWC Collaboration}

${ }^{a}$ Max Planck Institut für Kernphysik, Saupfercheckweg 1, Heidelberg, Germany

E-mail: pooja@mpi-hd.mpg.de

The Fermi bubbles are structures observed in gamma rays at $\mathrm{GeV}$ energies, emanating from the central region of our galaxy and extending up to $8.5 \mathrm{kpc}$ above and below the galactic plane. While initial studies showed a flat brightness across the entire structure, more recent work found a brightening at the base. We perform a template-based search for TeV signals from the northern Fermi bubble and just from the base of it in data from the High Altitude Water Cherenkov (HAWC) gamma-ray observatory. We employ a profile likelihood approach to calculate the significance and flux from the search regions. With no significant signal from the northern Fermi bubble and its base, we report new upper limits on the integral flux at $95 \%$ confidence level. Our integral flux upper limits for the northern Fermi bubble are more constraining than the previous limits reported by HAWC. Moreover, we present, for the first time, $\mathrm{TeV}$ limits pertaining to the base of the bubble which constitutes a more fair comparison to Fermi Large Area Telescope data points close to this particular region.

\footnotetext{
${ }^{*}$ Presenter
} 


\section{Introduction}

The High Altitude Water Cherenkov (HAWC) gamma-ray observatory is an air shower particle detector, located at an altitude of 4100 metres a.s.l., close to Pico de Orizaba, Mexico, with the coordinates being $97.3^{\circ} \mathrm{W}$ and $19.0^{\circ} \mathrm{N}$. HAWC was inaugurated in March 2015 and consists of an array of water tanks that function as water Cherenkov detectors designed to operate in the gammaray energy range of $300 \mathrm{GeV}$ to $100 \mathrm{TeV}$. It continuously surveys the sky overhead and is ideal for studying large-scale structures.

Some of the largest gamma-ray structures in our Milky Way galaxy are the Fermi bubbles. These are gamma-ray structures that appear to be emanating from the central part of the galaxy. They are tremendous in size, extending up to $55^{\circ}(\equiv 8 \mathrm{kpc}$ ) above and below the Galactic plane. Although initial reports have claimed a sharp cut-off in the spectrum of the Fermi bubbles and a flat projected brightness distribution [1,2], there is a gradient in the surface brightness profile of the bubbles [3]. A recent study [4] provides evidence of harder and brighter gamma-ray emission coming from the base of the bubble which fits a power-law spectrum without cut-off.

A search of very high energy gamma-ray signal from the northern Fermi bubble with HAWC was presented in 2017 [5]. With only 290 days of data, no significant excess was observed from the region corresponding to the northern Fermi bubble and differential upper limits above $1 \mathrm{TeV}$ were computed. We perform another search in a similar region with 800 days of HAWC data, new sets of gamma/hadron cuts, a more appropriate background model, and a different approach for computing significance [3]. The Fermi bubble excess is expected to be faint and distributed over an extended region. Therefore, we implement both a uniform and a weighted spatial template to perform a signal search from the northern Fermi bubble and just from its base. The uniform template for the northern Fermi bubble, henceforth NFB, was obtained from Fermi Tools and truncated at latitude $10^{\circ}$, as illustrated in Figure 1. In the following subsections, we describe how we derive the uniform template for the base for the northern Fermi bubble and the weighted spatial template for both regions.

\subsection{Deriving Uniform Spatial Template for the Base of the Northern Fermi Bubble}

The sensitivity of HAWC is dependent on declination, we therefore select a region which most likely contains the signal for the prominent part of the emission. We define a four-sided search region, henceforth referred to as the wedge, as illustrated in Figure 1, with the following boundaries:

1. $b=10^{\circ}$ : The region below this line is typically referred to as the base of the Fermi bubble;

2. $b=3^{\circ}$ : Limit to avoid most of Galactic plane emission;

3. $\delta=-25^{\circ}$ : Declination limit for HAWC field of view;

4. $b=10.5\left(\cosh \left(\frac{l-1}{10.5}\right)-1\right)$ : Bubble boundary obtained by comparing the Fermi Large Area Telescope (LAT) data to the X-ray edges [6]. 


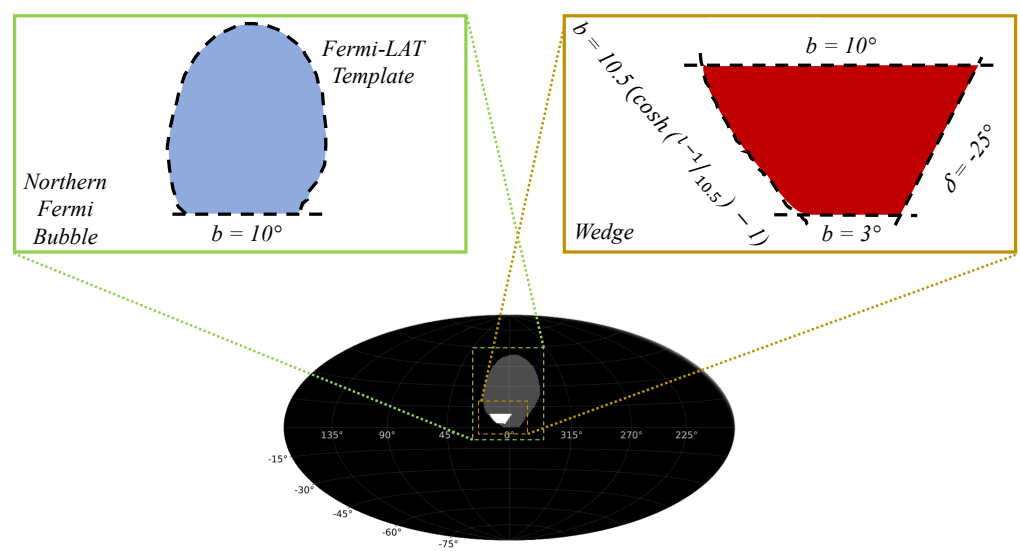

Figure 1: Description of search regions, illustrating the northern Fermi bubble (NFB) and the wedge at the base of the northern Fermi bubble.

\subsection{Modelling Weighted Spatial Template through Lon-Lat Surface Brightness Profiles}

We make use of the publicly available data ${ }^{1}$ from Fermi-LAT [7], which includes a residual counts map and its corresponding exposure map. The residual map comprises counts from the central part of the sky map with point sources masked out and consists primarily of the Fermi bubbles, inverse Compton (IC) emission, isotropic background and Loop I [8]. The exposure map consists of the expected counts from sources with respect to the amount of time that they spent in the field of view of the LAT instrument.

We select longitude and latitude regions on the exposure corrected residual map to extract corresponding surface brightness profiles. For latitude profile where $b>10^{\circ}$, displayed in blue in the right panel of Figure 2, we essentially sum the exposure corrected counts every $2^{\circ}$ and divide it by the solid angle that it occupies. For $b<10^{\circ}$, using $1^{\circ}$ strips, we extracted the counts for the wedge as well, displayed in red in the right panel of Figure 2. Since we do not have the original counts map but a modified residual one, we compute the standard error, i.e. root-mean-square of fluctuations about the average value, which we associate with each value extracted from the $2^{\circ}$ (or respectively, $1^{\circ}$ for the wedge) strip. From the data, it is evident that there is brighter emission from the base of the bubble and contrary to the initial result [2], there is a latitude dependence in the surface brightness profile. In principle, a continuous function should describe the surface brightness profile over the entire Fermi bubble. However, we choose to work with two distinct linear fits, which can be eventually improved upon if required.

We repeat the profile extraction and error computation for longitude. Here, we fit with a top hat function, as depicted in the left panel of Figure 2, where once more, the fit is not physically motivated but constitutes a simplified starting point which can be refined if required. By convolving the linear fits in latitude and the same top hat fit in longitude for $b<10^{\circ}$ as well as for $b>10^{\circ}$, and then projecting back to the Fermi bubble region, we retrieve a counts map analogous to the residual map that served as the starting point.

\footnotetext{
${ }^{1}$ https://www-glast.stanford.edu/pub_data/1220/GCexcess_Pass8_1704.03910_data/
} 


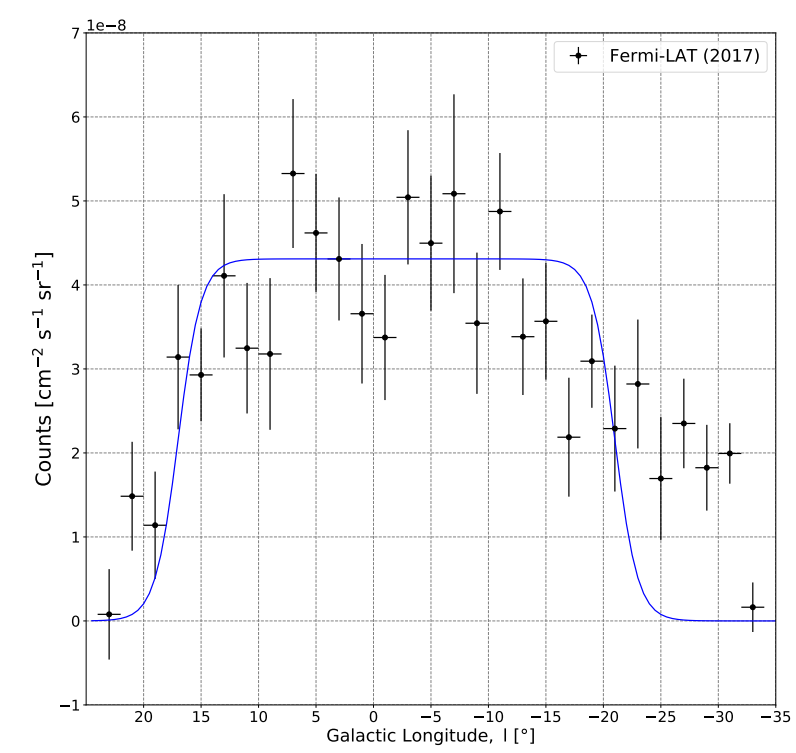

(a) Extracted longitude profile with Heaviside step functions used in top hat fit.

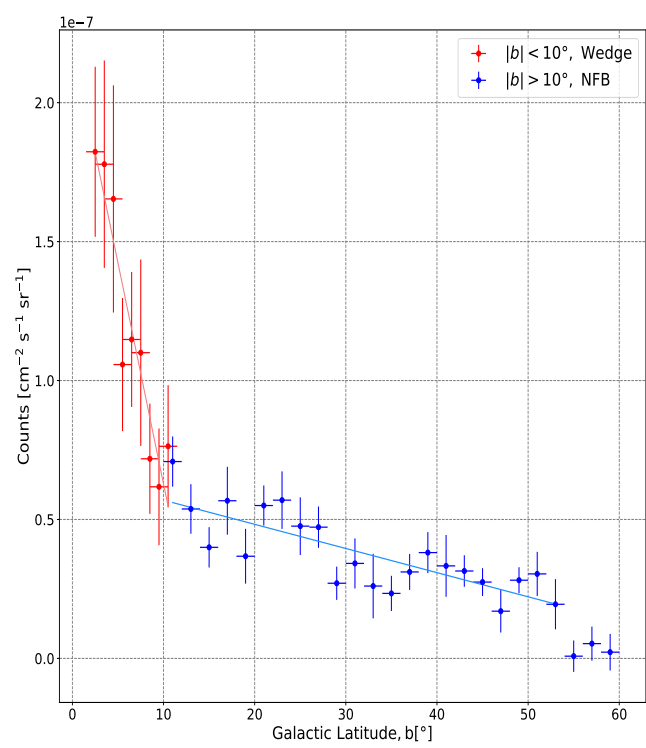

(b) Extracted latitude surface brightness profile and linear fits applied. A comparison between the wedge, in red, and the NFB, in blue.

Figure 2: Longitude and latitude surface brightness profiles for the northern Fermi bubble (NFB) and its base (wedge). Data points are exposure corrected counts per solid angle derived from [7].

We can verify the validity of the simplistic fits performed on the profiles by generating a difference map. This map is constructed by subtracting the counts map from the residual FermiLAT map. For visualisation purposes, we apply a $10^{\circ}$ smoothing, i.e. we integrate the counts in a $10^{\circ}$ radius for every pixel of both the residual Fermi-LAT map and the difference map as depicted in Figure 3. From the difference map, it is evident that our simplistic fits are adequate as they conveniently describe the northern Fermi bubble which could be excised. Now that we have a valid model for expected counts from different parts of the northern Fermi bubble, we normalise the sum of counts in the bubble to create a weighted spatial template of the NFB and the wedge.
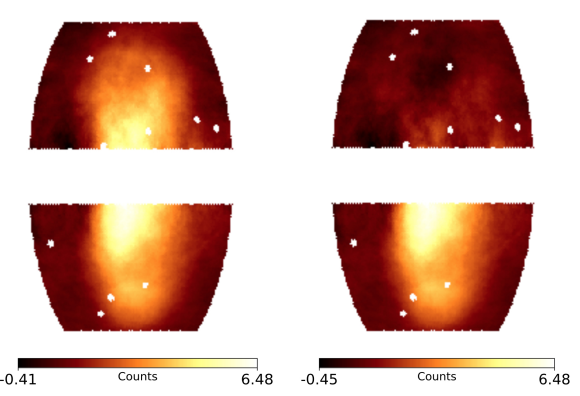

Figure 3: Spatial template evaluation. The left panel shows the residual counts map from Fermi-LAT [7] smoothed with a $10^{\circ}$ integration radius and the right panel shows a map with same smoothing after subtraction of the modelled counts from the northern Fermi bubble region. 


\section{Model Independent Flux Limits}

Using the profile likelihood approach described in [3], we search for an excess signal within the four aforementioned spatial templates. We compare our results for the base of the Fermi bubbles to those from [4] which are also for the base of the Fermi bubbles but not exactly the same as the wedge. Figure 4 illustrates the different regions, to scale, whose fluxes are compared.

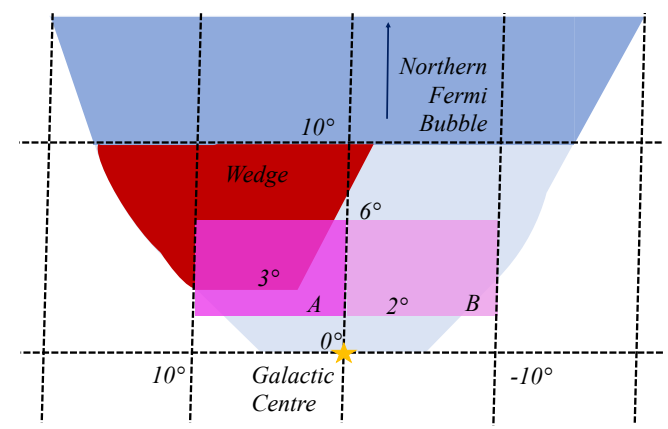

Figure 4: Description of regions being compared at the base of the Fermi bubble.

Figure 5 illustrates the observed spectrum and integral flux upper limits of the NFB and the wedge. Although we expected more emission from the wedge, we have a higher flux limit than for the $b>10^{\circ} \mathrm{NFB}$. This is due to the substantial decline in HAWC sensitivity at low declinations. We fit an exponential cut-off power-law such that it would satisfy both the Fermi-LAT observations [2] and (barely) our integral flux upper limit. From this fit, the slope $\Gamma$ is -2.25 and the cut-off energy occurs at $3.6 \mathrm{TeV}$ [3]. The relation between $E_{e}$ and $E_{\gamma}$ produced through IC scattering is $E_{e} \approx 11 \sqrt{E_{\gamma}} \mathrm{TeV}$. Hence, in a leptonic scenario for gamma-ray emission, we expect electrons with cut-off energy $\sim 21 \mathrm{TeV}$ from the northern Fermi bubble. For gamma rays produced via hadronic interactions, the energy of the proton and photon energies are related as $E_{p} \approx 23.6 E_{\gamma} \mathrm{TeV}$ [9], with an expected proton cut-off at $\sim 85 \mathrm{TeV}$ [3].

To grasp the implications of this high-energy proton cut-off, we depict, in Figure 6, the acceleration timescale for second-order Fermi acceleration of interacting magnetohydrodynamic (MHD) turbulence with Alfvén waves and the escape timescale through diffusion in the Kolmogorov limit. A more detailed description of these timescales can be found in $[3,10]$. For this phase-space plot, we varied two parameters: density and magnetic field. We find that a maximum density of $10^{-2} \mathrm{~cm}^{-3}$ and a minimum magnetic field of $7 \mu \mathrm{G}$ satisfy the hadronic upper limit where protons accelerated up to $\sim 85 \mathrm{TeV}$ are confined and undergo $p p$-interaction while those accelerated above $\sim 85 \mathrm{TeV}$ escape the bubble [3]. Densities above $10^{-2} \mathrm{~cm}^{-3}$ would conflict with proton-proton ( $p p$ ) losses. This density and magnetic field are also in agreement with other studies, for instance, [11] and [12].

\section{Conclusions}

The Fermi bubbles are few kpc-scale structures that appear to be originating from the central region of our Milky Way galaxy. The physical mechanisms that source the bubbles and produce 


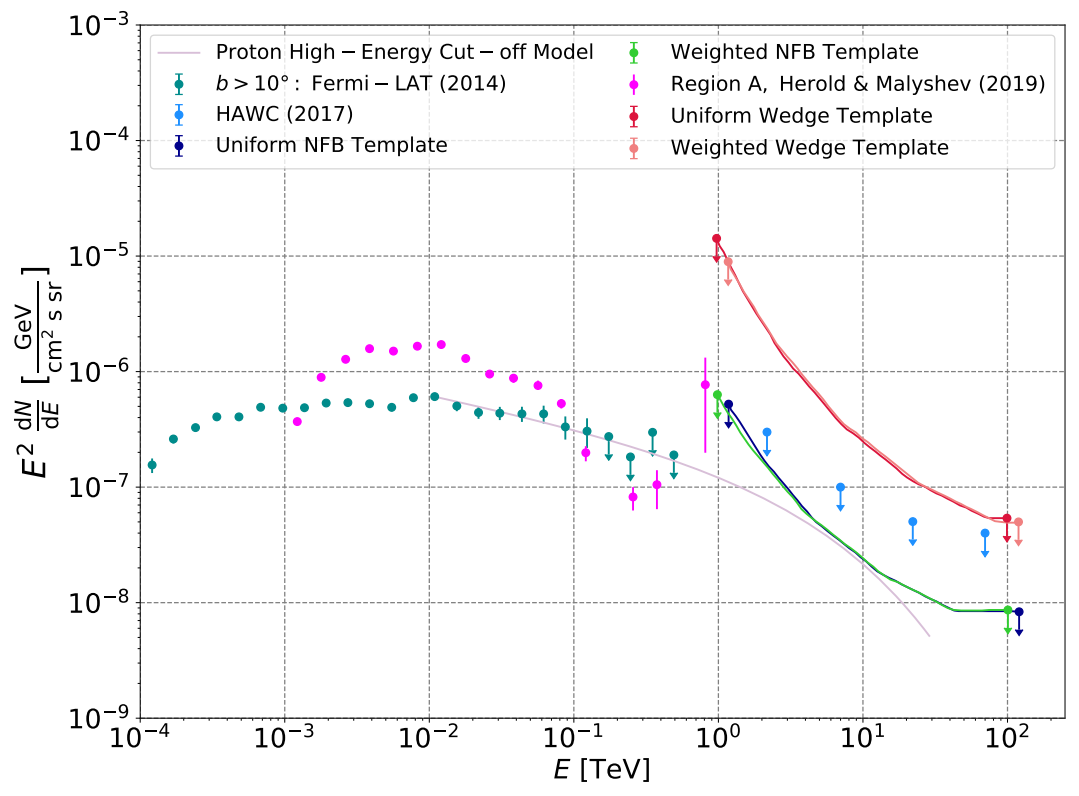

Figure 5: Flux upper limit (at 95\% confidence level) comparison between northern Fermi bubble (NFB) and wedge, at the base of the bubble. A probable proton high-energy cut-off model, extending to TeV energies, is shown.

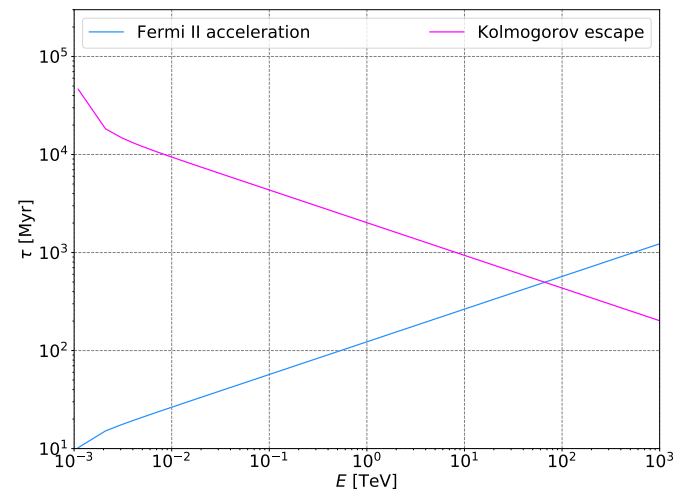

Figure 6: Interaction timescales of Fermi second-order acceleration and escape through Kolmogorov diffusion for protons.

the observed spectrum are still elusive. Two of the leading gamma-ray production mechanisms involve leptonic or hadronic scenarios, both of which fit the hard gamma-ray spectrum observed and energetics involved but none of them alone can explain all of the observed features and the associated substructures. We performed a template-based search for $\mathrm{TeV}$ signals from the northern Fermi bubble and just from the base of it. With no significant excess observed, which could be due to the substantial decline in HAWC sensitivity at low declination, we computed integral upper limits at $95 \%$ confidence level.

Our integral flux upper limits for the northern Fermi bubble are more constraining than the 
previous limits reported by HAWC while those for the base region provide a more fair comparison to Fermi-LAT data points from the base of the bubble. For the northern Fermi bubble, we present a hadronic model with proton cut-off energy at $85 \mathrm{TeV}$ that fits the Fermi-LAT data and conforms to our flux limits. This fit is a power-law with index -2.25 and cut-off at $3.6 \mathrm{TeV}$ [3]. Using the high-energy proton cut-off, we further constrain the density and magnetic field of the bubble to be $\sim 10^{-2} \mathrm{~cm}^{-3}$ and $\sim 7 \mu \mathrm{G}$, respectively [3].

\section{Acknowledgements}

We acknowledge the support from: the US National Science Foundation (NSF); the US Department of Energy Office of High-Energy Physics; the Laboratory Directed Research and Development (LDRD) program of Los Alamos National Laboratory; Consejo Nacional de Ciencia y Tecnología (CONACyT), México, grants 271051, 232656, 260378, 179588, 254964, 258865, 243290, 132197, A1-S-46288, A1-S-22784, cátedras 873, 1563, 341, 323, Red HAWC, México; DGAPA-UNAM grants IG101320, IN111716-3, IN111419, IA102019, IN110621, IN110521; VIEP-BUAP; PIFI 2012, 2013, PROFOCIE 2014, 2015; the University of Wisconsin Alumni Research Foundation; the Institute of Geophysics, Planetary Physics, and Signatures at Los Alamos National Laboratory; Polish Science Centre grant, DEC-2017/27/B/ST9/02272; Coordinación de la Investigación Científica de la Universidad Michoacana; Royal Society - Newton Advanced Fellowship 180385; Generalitat Valenciana, grant CIDEGENT/2018/034; Chulalongkorn University's CUniverse (CUAASC) grant; Coordinación General Académica e Innovación (CGAI-UdeG), PRODEP-SEP UDG-CA499; Institute of Cosmic Ray Research (ICRR), University of Tokyo, H.F. acknowledges support by NASA under award number 80GSFC21M0002. We also acknowledge the significant contributions over many years of Stefan Westerhoff, Gaurang Yodh and Arnulfo Zepeda Dominguez, all deceased members of the HAWC collaboration. Thanks to Scott Delay, Luciano Díaz and Eduardo Murrieta for technical support.

\section{References}

[1] M. Su, T.R. Slatyer and D.P. Finkbeiner, Giant Gamma-ray Bubbles from Fermi-LAT: Active Galactic Nucleus Activity or Bipolar Galactic Wind?, ApJ 724 (2010) 1044 [1005 . 5480].

[2] Fermi-LAT Collaboration, M. Ackermann, A. Albert, W.B. Atwood, L. Baldini, J. Ballet et al., The Spectrum and Morphology of the Fermi Bubbles, ApJ 793 (2014) 64 [1407 . 7905].

[3] P. Surajbali, Observing large-scale structures in the gamma-ray sky, Ph.D. thesis, Ruperto-Carola University of Heidelberg, July, 2020.

[4] L. Herold and D. Malyshev, Hard and bright gamma-ray emission at the base of the Fermi bubbles, A\&A 625 (2019) A110 [1904.01454].

[5] HAWC Collaboration, A.U. Abeysekara, A. Albert, R. Alfaro, C. Alvarez, J.D. Álvarez et al., Search for Very High-energy Gamma Rays from the Northern Fermi Bubble Region with HAWC, ApJ 842 (2017) 85 [1703.01344]. 
[6] J.-M. Casandjian, The Fermi-LAT model of interstellar emission for standard point source analysis, arXiv e-prints (2015) arXiv: 1502.07210 [1502 . 07210].

[7] Fermi LAT Collaboration, M. Ackermann, M. Ajello, A. Albert, W.B. Atwood, L. Baldini et al., The Fermi Galactic Center GeV Excess and Implications for Dark Matter, ApJ 840 (2017) 43 [1704.03910].

[8] M.I. Large, M.J.S. Quigley and C.G.T. Haslam, A new feature of the radio sky, MNRAS 124 (1962) 405.

[9] A. Kappes, J. Hinton, C. Stegmann and F.A. Aharonian, Potential Neutrino Signals from Galactic $\gamma$-Ray Sources, ApJ 656 (2007) 870 [astro-ph/0607286].

[10] R. Schlickeiser, Cosmic Ray Astrophysics, Springer Science \& Business Media, Berlin (2002).

[11] Y. Fujita, Y. Ohira and R. Yamazaki, A Hadronic-leptonic Model for the Fermi Bubbles: Cosmic-Rays in the Galactic Halo and Radio Emission, ApJ 789 (2014) 67 [1405 . 5214].

[12] Fermi-LAT Collaboration, M. Ackermann, M. Ajello, K. Asano, W.B. Atwood, M. Axelsson et al., Fermi-LAT Observations of the Gamma-Ray Burst GRB 130427A, Science 343 (2014) 42 [1311. 5623]. 


\section{Full Authors List: HAWC Collaboration}

A.U. Abeysekara ${ }^{48}$, A. Albert $^{21}$, R. Alfaro ${ }^{14}$, C. Alvarez $^{41}$, J.D. Álvarez ${ }^{40}$, J.R. Angeles Camacho ${ }^{14}$, J.C. Arteaga-Velázquez ${ }^{40}$, K. P. Arunbabu $^{17}$, D. Avila Rojas ${ }^{14}$, H.A. Ayala Solares ${ }^{28}$, R. Babu ${ }^{25}$, V. Baghmanyan ${ }^{15}$, A.S. Barber ${ }^{48}$, J. Becerra Gonzalez ${ }^{11}$, E. BelmontMoreno $^{14}$, S.Y. BenZvi ${ }^{29}$, D. Berley ${ }^{39}$, C. Brisbois ${ }^{39}$, K.S. Caballero-Mora ${ }^{41}$, T. Capistrán ${ }^{12}$, A. Carramiñana ${ }^{18}$, S. Casanova ${ }^{15}$, O. Chaparro-Amaro $^{3}$, U. Cotti ${ }^{40}$, J. Cotzomi ${ }^{8}$, S. Coutiño de León ${ }^{18}$, E. De la Fuente ${ }^{46}$, C. de León ${ }^{40}$, L. Diaz-Cruz ${ }^{8}$, R. Diaz Hernandez ${ }^{18}$, J.C. Díaz-Vélez ${ }^{46}$, B.L. Dingus ${ }^{21}$, M. Durocher ${ }^{21}$, M.A. DuVernois ${ }^{45}$, R.W. Ellsworth ${ }^{39}$, K. Engel ${ }^{39}$, C. Espinoza ${ }^{14}$, K.L. Fan ${ }^{39}$, K. Fang $^{45}$, M. Fernández Alonso ${ }^{28}$, B. Fick ${ }^{25}$, H. Fleischhack ${ }^{51,11,52}$, J.L. Flores ${ }^{46}$, N.I. Fraija ${ }^{12}$, D. Garcia ${ }^{4}$, J.A. García-González ${ }^{20}$, J. L. García-Luna ${ }^{46}$, G. García-Torales ${ }^{46}$, F. Garfias ${ }^{12}$, G. Giacinti ${ }^{22}$, H. Goksu ${ }^{22}$, M.M. González ${ }^{12}$, J.A. Goodman ${ }^{39}$, J.P. Harding ${ }^{21}$, S. Hernandez ${ }^{14}$, I. Herzog ${ }^{25}$, J. Hinton ${ }^{22}$, B. Hona ${ }^{48}$, D. Huang ${ }^{25}$, F. Hueyotl-Zahuantitla ${ }^{41}$, C.M. Hui ${ }^{23}$, B. Humensky ${ }^{39}$, P. Hüntemeyer ${ }^{25}$, A. Iriarte ${ }^{12}$, A. Jardin-Blicq ${ }^{22,49,50}$, H. Jhee ${ }^{43}$, V. Joshi ${ }^{7}$, D. Kieda ${ }^{48}$, G J. Kunde ${ }^{21}$, S. Kunwar ${ }^{22}$, A. Lara ${ }^{17}$, J. Lee ${ }^{43}$, W.H. Lee ${ }^{12}$, D. Lennarz ${ }^{9}$, H. León Vargas ${ }^{14}$, J. Linnemann ${ }^{24}$, A.L. Longinotti ${ }^{12}$, R. López-Coto ${ }^{19}$, G. Luis-Raya ${ }^{44}$, J. Lundeen ${ }^{24}$, K. Malone ${ }^{21}$, V. Marandon $^{22}$, O. Martinez ${ }^{8}$, I. Martinez-Castellanos ${ }^{39}$, H. Martínez-Huerta ${ }^{38}$, J. Martínez-Castro ${ }^{3}$, J.A.J. Matthews ${ }^{42}$, J. McEnery ${ }^{11}$, P. Miranda-Romagnoli $^{34}$, J.A. Morales-Soto ${ }^{40}$, E. Moreno ${ }^{8}$, M. Mostafá ${ }^{28}$, A. Nayerhoda ${ }^{15}$, L. Nellen ${ }^{13}$, M. Newbold ${ }^{48}$, M.U. Nisa ${ }^{24}$, R. Noriega-Papaqui ${ }^{34}$, L. Olivera-Nieto ${ }^{22}$, N. Omodei ${ }^{32}$, A. Peisker ${ }^{24}$, Y. Pérez Araujo ${ }^{12}$, E.G. Pérez-Pérez ${ }^{44}$, C.D. Rho ${ }^{43}$, C. Rivière ${ }^{39}$, D. Rosa-Gonzalez ${ }^{18}$, E. Ruiz-Velasco ${ }^{22}$, J. Ryan ${ }^{26}$, H. Salazar ${ }^{8}$, F. Salesa Greus ${ }^{15,53}$, A. Sandoval ${ }^{14}$, M. Schneider ${ }^{39}$, H. Schoorlemmer ${ }^{22}$, J. Serna-Franco ${ }^{14}$, G. Sinnis ${ }^{21}$, A.J. Smith ${ }^{39}$, R.W. Springer ${ }^{48}$, P. Surajbali ${ }^{22}$, I. Taboada ${ }^{9}$, M. Tanner ${ }^{28}$, K. Tollefson ${ }^{24}$, I. Torres ${ }^{18}$, R. Torres-Escobedo $^{30}$, R. Turner ${ }^{25}$, F. Ureña-Mena ${ }^{18}$, L. Villaseñor ${ }^{8}$, X. Wang ${ }^{25}$, I.J. Watson ${ }^{43}$, T. Weisgarber ${ }^{45}$, F. Werner ${ }^{22}$, E. Willox ${ }^{39}$, J. Wood ${ }^{23}$, G.B. Yodh ${ }^{35}$, A. Zepeda ${ }^{4}$, H. Zhou ${ }^{30}$

${ }^{1}$ Barnard College, New York, NY, USA, ${ }^{2}$ Department of Chemistry and Physics, California University of Pennsylvania, California, PA, USA, ${ }^{3}$ Centro de Investigación en Computación, Instituto Politécnico Nacional, Ciudad de México, México, ${ }^{4}$ Physics Department, Centro de Investigación y de Estudios Avanzados del IPN, Ciudad de México, México, ${ }^{5}$ Colorado State University, Physics Dept., Fort Collins, CO, USA, ${ }^{6}$ DCI-UDG, Leon, Gto, México, ${ }^{7}$ Erlangen Centre for Astroparticle Physics, Friedrich Alexander Universität, Erlangen, BY, Germany, ${ }^{8}$ Facultad de Ciencias Físico Matemáticas, Benemérita Universidad Autónoma de Puebla, Puebla, México, ${ }^{9}$ School of Physics and Center for Relativistic Astrophysics, Georgia Institute of Technology, Atlanta, GA, USA, ${ }^{10}$ School of Physics Astronomy and Computational Sciences, George Mason University, Fairfax, VA, USA, ${ }^{11}$ NASA Goddard Space Flight Center, Greenbelt, MD, USA, ${ }^{12}$ Instituto de Astronomía, Universidad Nacional Autónoma de México, Ciudad de México, México, ${ }^{13}$ Instituto de Ciencias Nucleares, Universidad Nacional Autónoma de México, Ciudad de México, México, ${ }^{14}$ Instituto de Física, Universidad Nacional Autónoma de México, Ciudad de México, México, ${ }^{15}$ Institute of Nuclear Physics, Polish Academy of Sciences, Krakow, Poland, ${ }^{16}$ Instituto de Física de São Carlos, Universidade de São Paulo, São Carlos, SP, Brasil, ${ }^{17}$ Instituto de Geofísica, Universidad Nacional Autónoma de México, Ciudad de México, México, ${ }^{18}$ Instituto Nacional de Astrofísica, Óptica y Electrónica, Tonantzintla, Puebla, México, ${ }^{19}$ INFN Padova, Padova, Italy, ${ }^{20}$ Tecnologico de Monterrey, Escuela de Ingeniería y Ciencias, Ave. Eugenio Garza Sada 2501, Monterrey, N.L., 64849, México, ${ }^{21}$ Physics Division, Los Alamos National Laboratory, Los Alamos, NM, USA, ${ }^{22}$ Max-Planck Institute for Nuclear Physics, Heidelberg, Germany, ${ }^{23}$ NASA Marshall Space Flight Center, Astrophysics Office, Huntsville, AL, USA, ${ }^{24}$ Department of Physics and Astronomy, Michigan State University, East Lansing, MI, USA, ${ }^{25}$ Department of Physics, Michigan Technological University, Houghton, MI, USA, ${ }^{26}$ Space Science Center, University of New Hampshire, Durham, NH, USA, ${ }^{27}$ The Ohio State University at Lima, Lima, OH, USA, ${ }^{28}$ Department of Physics, Pennsylvania State University, University Park, PA, USA, ${ }^{29}$ Department of Physics and Astronomy, University of Rochester, Rochester, NY, USA, ${ }^{30}$ Tsung-Dao Lee Institute and School of Physics and Astronomy, Shanghai Jiao Tong University, Shanghai, China, ${ }^{31}$ Sungkyunkwan University, Gyeonggi, Rep. of Korea, ${ }^{32}$ Stanford University, Stanford, CA, USA, ${ }^{33}$ Department of Physics and Astronomy, University of Alabama, Tuscaloosa, AL, USA, ${ }^{34}$ Universidad Autónoma del Estado de Hidalgo, Pachuca, Hgo., México, ${ }^{35}$ Department of Physics and Astronomy, University of California, Irvine, Irvine, CA, USA, ${ }^{36}$ Santa Cruz Institute for Particle Physics, University of California, Santa Cruz, Santa Cruz, CA, USA, ${ }^{37}$ Universidad de Costa Rica, San José, Costa Rica, ${ }^{38}$ Department of Physics and Mathematics, Universidad de Monterrey, San Pedro Garza García, N.L., México, ${ }^{39}$ Department of Physics, University of Maryland, College Park, MD, USA, ${ }^{40}$ Instituto de Física y Matemáticas, Universidad Michoacana de San Nicolás de Hidalgo, Morelia, Michoacán, México, ${ }^{41}$ FCFM-MCTP, Universidad Autónoma de Chiapas, Tuxtla Gutiérrez, Chiapas, México, ${ }^{42}$ Department of Physics and Astronomy, University of New Mexico, Albuquerque, NM, USA, ${ }^{43}$ University of Seoul, Seoul, Rep. of Korea, ${ }^{44}$ Universidad Politécnica de Pachuca, Pachuca, Hgo, México, ${ }^{45}$ Department of Physics, University of Wisconsin-Madison, Madison, WI, USA, ${ }^{46}$ CUCEI, CUCEA, Universidad de Guadalajara, Guadalajara, Jalisco, México, ${ }^{47}$ Universität Würzburg, Institute for Theoretical Physics and Astrophysics, Würzburg, Germany, ${ }^{48}$ Department of Physics and Astronomy, University of Utah, Salt Lake City, UT, USA, ${ }^{49}$ Department of Physics, Faculty of Science, Chulalongkorn University, Pathumwan, Bangkok 10330, Thailand, ${ }^{50}$ National Astronomical Research Institute of Thailand (Public Organization), Don Kaeo, MaeRim, Chiang Mai 50180, Thailand, ${ }^{51}$ Department of Physics, Catholic University of America, Washington, DC, USA, ${ }^{52}$ Center for Research and Exploration in Space Science and Technology, NASA/GSFC, Greenbelt, MD, USA, ${ }^{53}$ Instituto de Física Corpuscular, CSIC, Universitat de València, Paterna, Valencia, Spain 